\title{
Pricing and Warranty Decisions of Substitutable Products for a Fuzzy Two-Echelon Supply Chain
}

\author{
Yongzhao Wang \\ School of Mathematics and Statistics, Anyang Normal University, Xuange Avenue 436, Anyang, China \\ Correspondence should be addressed to Yongzhao Wang; wangyongzhao1987@126.com
}

Received 21 October 2016; Accepted 12 December 2016; Published 16 January 2017

Academic Editor: Paolo Renna

Copyright (C) 2017 Yongzhao Wang. This is an open access article distributed under the Creative Commons Attribution License, which permits unrestricted use, distribution, and reproduction in any medium, provided the original work is properly cited.

\begin{abstract}
This paper considers the optimal decisions of pricing and warranty level for substitutable products in a fuzzy supply chain environment, where the consumer demands, manufacturing costs, and warranty costs are characterized as fuzzy variables. Expected value manufacturer-leader Stackelberg models and two chance-constrained programming models, that is, the $\lambda$-optimistic and $\lambda$ pessimistic manufacturer-leader Stackelberg models, are established. The corresponding closed-form solutions are obtained by using the game-theoretical approach and fuzzy theory. Finally, numerical examples are presented to illustrate the effectiveness of the models and to provide managerial insights for decision makers. The results show that it would be beneficial for the whole supply chain when the warranty services are introduced into the market, and the firm which provides the warranty service has the advantage in holding more dominant position.
\end{abstract}

\section{Introduction}

Price is the most important and direct criterion that consumers use to evaluate a product at the market. With the technological advancements accelerated, which have shortened product life cycles and intensified business competition. More and more firms have improved their market competitiveness by improving services and/or product quality, so as to build brand loyalty to avoid traditional competition which focuses solely on price. For instance, IBM and Dell have a good reputation with dependence on their customer services and supports [1]. Therefore, warranty has become an effective measure for encouraging market demand by reducing risks for consumers and has also associated with the firms' corporate social responsibility which is a key characteristic of corporate sustainability and business sustainability (Kimble and Lesher [2], Van Marrewijk [3], Ahi and Searcy [4], and Jeyakumar et al. [5]). A warranty represents the commitment for producers to restore the functionalities of faulty product within a certain period after it is sold. The majority of consumers prefer to purchase a product from a manufacturer with a warranty ensuring the replacement or repairing of the product during the warranty period, with little or no charge [6], which can also ensure an increasing of sales leading to more profits for the supply-chain members.

Warranty has received extensive attention in literature. Chung and Wee [7] considered the pricing policy, imperfect production, inspection planning, warranty period, and the stock-level-dependent demand with the Weibull deterioration, partial backorder, and inflation and investigated an integrated production-inventory-deteriorating model. Wu et al. [8] developed a decision model for producers in the static demand market to determine the optimal price, warranty length, and production rate of a product to maximize profit based on the predetermined life cycle. Chen et al. [9] discussed the issues associated with a manufacturer's pricing strategies in a supply chain that comprises one manufacturer and two competing retailers, with warranty perioddependent demands. Wei et al. [1] explored the optimal pricing and warranty period decisions of two complementary products in a supply chain from a two-stage game theoretic perspective, where there are two manufacturers and one common retailer, such as Lin and Shue [10], Wu et al. [11], Tsao et al. [12], and Huang et al. [13].

Recently, several powerful retail chains have emerged around the world (Walmart, Carrefour, etc.). These powerful 
TABLE 1: Pricing strategies in the fuzzy environment.

\begin{tabular}{|c|c|c|c|}
\hline Paper & Decisions & Product status & Supply chain structure \\
\hline Gu and Ji [32] & $\begin{array}{l}\text { Pricing policy; } \\
\text { recycling }\end{array}$ & Single & One manufacturer, one retailer \\
\hline Zhou et al. [17] & Pricing policy & Single & One manufacturer, one retailer \\
\hline Guo and Liu [27] & $\begin{array}{l}\text { Pricing decision; } \\
\text { collaboration }\end{array}$ & Single & One manufacturer, one retailer \\
\hline Wei and Zhao [28] & $\begin{array}{c}\text { Pricing policy; } \\
\text { remanufacturing rate }\end{array}$ & Single & One manufacturer, two retailers \\
\hline Zhao et al. [29] & Pricing policy & Substitutable & One manufacturer, two retailers \\
\hline Zhao et al. [14] & $\begin{array}{l}\text { Pricing policy; } \\
\text { service level }\end{array}$ & Substitutable & Two manufacturers, one retailer \\
\hline Wei et al. [30] & Pricing policy & Single & One manufacturer, duopolistic retailers \\
\hline Song et al. [23] & $\begin{array}{l}\text { Pricing policy; } \\
\text { selling effort }\end{array}$ & Single & One manufacturer, one retailer \\
\hline Wang et al. [31] & Pricing policy & Complementary & Two manufacturers \\
\hline Zhao and Wang [24] & $\begin{array}{l}\text { Pricing policy; } \\
\text { service level }\end{array}$ & Single & One manufacturer, two retailers \\
\hline
\end{tabular}

retailers have a dominant position relative to most manufacturers on the market, and multiple substitutable products of an item sourced from two different manufacturers will appear on Walmart's shelves at the same time. For instance, Walmart retails substitute brands of detergents like Unilever brands (Surf, Wisk) and P\&G brands (Tide, Gain, and Cheer) [14]. The influence of these powerful retailers continues to expand on the market, and their perspective of channel problems attracts more and more attention of scholars $[15,16]$.

In order to make effective supply chain strategies, we cannot ignore the uncertainties that happen in the real world. And those uncertainties are usually associated with the product supply, the customer demand, the manufacturing cost, and so on [17-19]. More recently, predecessors provided a new foundation for optimization problems in the fuzzy environment, in which various models such as the expected value and the chance-constrained programming were proposed to deal with optimization problems (Dubois and Prade [20]; Liu [21, 22]). In recent supply chain studies, Zhou et al. [17] established the expected value models as well as chance-constrained programming models to determine the pricing strategies for the retailer and the manufacturer. Song et al. [23] discussed the optimal decisions of pricing and selling effort for a two-echelon supply chain with uncertain consumer demands, manufacturing costs, and selling costs. Zhao and Wang [24] studied the pricing and retail service decisions of three different game structure models in a supply chain which was assumed in a fuzzy uncertainty environment. Zhao and Wei [25], Chakraborty et al. [26], and so on. Table 1 illustrates some recent papers on pricing strategies within supply chain in the fuzzy environment as follows [14, 17, 23, 24, 27-31].

Differing from those of prior studies, by considering a fuzzy supply chain where two manufacturers produce two substitutable products and sell them to a retailer who sells them to the end consumers, this paper studies the optimal wholesale prices, warranty levels, and retail prices' decisions. The main contributions of this paper are as follows: first, we establish one expected value MS model and two chance-constrained programming models (i.e., $\lambda$-optimistic and $\lambda$-pessimistic). Second, by using the game-theoretical approach and fuzzy theory, the optimal wholesale prices, warranty levels, and retail prices are obtained in the three decision models. Third, through numerical studies, we get some managerial insights: (1) the firm's optimal pricing and warranty strategy and the corresponding profits vary with the ranking measure. The strategy in the $\lambda$-optimistic Stackelberg model is always the highest, and the strategy in the $\lambda$ pessimistic Stackelberg model is always the lowest. (2) It would be beneficial for the whole supply chain when the warranty services are introduced into the market. The firm which provides the warranty service has the advantage in holding more dominant position and so on.

The rest of the paper is organized as follows. The problem description and notations are presented in Section 2. In Section 3, we formulate the expected value model and two chance-constrained programming MS Stackelberg models with fuzzy consumer's demands, fuzzy manufacturer costs, and fuzzy warranty costs. Section 4 gives some numerical examples to illustrate the effectiveness of the models. A conclusion is presented in Section 5.

\section{Problem Description}

In our symmetric-information two-echelon supply chain structure in a fuzzy environment, there are two competitive manufactures (indexed by $m_{1}, m_{2}$ ) and one common retailer. The manufacturer $m_{i}$ produces a substitutable product with manufacturing $\operatorname{cost} c_{i}$ and wholesales it with wholesale price $w_{i}$ to the common retailer, who in turn sells it to the 
consumer with retail price $p_{i}(i=1,2)$, respectively. The two manufacturers provide warranty $t_{1}$ and $t_{2}$ to the retailer.

We assume all activity occurs within a single period and ignore the logistic cost components of each firm for analytical convenience. Further, the two manufacturers hold more market power than the common retailer in the market, and the manufacturers act as the Stackelberg leader. Under such a scenario, the two manufacturers and common retailer make their pricing and warranty decisions in order to achieve maximal expected profits.

The consumer's demand is sensitive to the retail prices and the manufacturer warranty levels. Similar to Wang and Zhao [33] and Wei et al. [1], we define the consumer's demand function as a linear form in this paper, which is decreasing in its own price and increasing in the opponent's price, moreover, increasing in its own warranty level and decreasing in the opponent's warranty level.

$$
\begin{aligned}
& D_{i}\left(p_{1}, p_{2}, t_{1}, t_{2}\right)=\alpha_{i}-\beta_{1} p_{i}+\beta_{2} p_{j}+\gamma_{1} t_{i}-\gamma_{2} t_{j}, \\
& i=1,2, j=3-i .
\end{aligned}
$$

The parameter $\alpha_{i}$ represents the primary market base of the product $i$. The parameters $\beta_{i}$ and $\gamma_{i}$ are price and warranty elastic coefficients, which measure the effectiveness of the retail prices and manufacturer's warranties in stimulating or restraining product's consumer demand, respectively. Moreover, we characterize the total cost of providing the warranty by $g(t)$, which is a strictly convex function and has been property of $\partial g(t) / \partial t>0$ and $\partial^{2} g(t) / \partial t^{2}>0$. Consistent with the extant literature (Xiao and Yang [34], etc.), we assume that the total cost of providing the warranty is $g_{i}(t)=l_{i} t_{i}^{2}$, where $l_{i}$ denotes the warranty cost coefficient of manufacturer $m_{i}$, $i=1,2$.

Since the decision makers have little or no historical data on consumer's demand, it is more appropriate to estimate the manufacturing cost and warranty cost coefficient based on decision makes judgements, intuitions, and experience. In this paper, we assume the parameters $c_{i}, \alpha_{i}, \beta_{i}, \gamma_{i}$, and $l_{i}$ are mutually independent nonnegative fuzzy parameters. The parameters $\beta_{i}$ and $\gamma_{i}$ satisfy $E\left[\beta_{1}\right]>E\left[\beta_{2}\right]$ and $E\left[\gamma_{1}\right]>$
$E\left[\gamma_{2}\right]$, which signify that the expected demand should be more sensitive to the changes in its own retail price and warranty than to the changes in the competitor's. The unit marginal profit $p_{i}-w_{i}$ and demand $D_{i}\left(p_{1}, p_{2}, t_{1}, t_{2}\right)$ are nonnegative in the real world; thus $\operatorname{Pos}\left\{p_{i}-c_{i}<0\right\}=0$ and $\operatorname{Pos}\left\{D_{i}\left(p_{1}, p_{2}, t_{1}, t_{2}\right)<0\right\}=0(i=1,2)$.

According to the problem descriptions and assumptions, the manufacturer $m_{i}$ and the retailer's profit functions can be expressed as follows:

$$
\begin{array}{ll}
\Pi_{m_{i}}\left(w_{i}, t_{i}\right)=\left(w_{i}-c_{i}\right) D_{i}\left(p_{1}, p_{2}, t_{1}, t_{2}\right)-l_{i} t_{i}^{2}, & i=1,2, \\
\Pi_{r}\left(p_{1}, p_{2}\right)=\sum_{i=1}^{2}\left(p_{i}-w_{i}\right) D_{i}\left(p_{1}, p_{2}, t_{1}, t_{2}\right) . &
\end{array}
$$

We impose the following conditions on the parameters to ensure that the profit expressions will be well behaved and possess a unique optimum: $8 \beta_{1 \lambda}^{L} l_{i \lambda}^{L}-\left(\gamma_{1 \lambda}^{U}\right)^{2}>0$ and $\left(\beta_{1 \lambda}^{L}\right)^{2}-$ $\left(\beta_{2 \lambda}^{U}\right)^{2}>0$.

\section{Fuzzy Models and Main Results}

In this section, we consider a scenario that the sizes of the two manufacturers are larger compared to the common retailer's in the market. The two manufacturers act as the Stackelberg leaders and the common retailer acts as the Stackelberg follower. Using the game-theoretical approach to analyze the model, we can get the timing of the game as follows: the manufacturers first choose the wholesale prices and the warranty levels of the product, simultaneously; after observing the wholesale prices and the warranty levels, the retailer then determines his retail prices of two products.

3.1. The $\lambda$-Optimistic Manufacturer-Leader Stackelberg (MSU) Model. With a given condition level $\lambda \in(0,1]$, we formulate the maximum chance-constrained programming model for the manufacturer-leader supply chain as follows:

$$
\begin{array}{ll}
\max _{w_{1}, t_{1}} & \bar{\Pi}_{m_{1}}\left(w_{1}\right) \\
\max _{w_{2}, t_{2}} & \bar{\Pi}_{m_{2}}\left(w_{2}\right) \\
\text { s.t. } & \operatorname{Pos}\left\{\left(w_{i}-c_{i}\right)\left(\alpha_{i}-\beta_{1} p_{i}^{*}\left(w_{i}, t_{i}, t_{j}\right)+\beta_{2} p_{j}^{*}\left(w_{j}, t_{j}, t_{i}\right)+\gamma_{1} t_{i}-\gamma_{2} t_{j}\right) \geq \bar{\Pi}_{m_{i}}\right\} \geq \lambda, \\
& \operatorname{Pos}\left\{w_{i}-c_{i}<0\right\}=0, \quad i=1,2, j=3-i . \\
& p_{1}^{*}, p_{2}^{*} \text { are derived from solving the problem } \\
& \begin{cases}\max _{p_{1}, p_{2}} \quad \bar{\Pi}_{r}\left(p_{1}, p_{2}\right) \\
\operatorname{s.t.} \quad & \operatorname{Pos}\left\{\left(p_{i}-w_{i}\right)\left(\alpha_{i}-\beta_{1} p_{i}+\beta_{2} p_{j}+\gamma_{1} t_{i}-\gamma_{2} t_{j}\right) \geq \bar{\Pi}_{r}\right\} \geq \lambda, \\
& \operatorname{Pos}\left\{\alpha_{i}-\beta_{1} p_{i}+\beta_{2} p_{j}+\gamma_{1} t_{i}-\gamma_{2} t_{j}<0\right\}=0, \\
& p_{i} \geq w_{i}, \quad i=1,2, j=3-i,\end{cases}
\end{array}
$$


where $\bar{\Pi}_{m_{i}}$ and $\bar{\Pi}_{r}$ are the threshold values of the manufacturers and the retailer; namely, $\bar{\Pi}_{m_{i}}$ and $\bar{\Pi}_{r}$ should be the maximum values that supply chain members' profit functions achieve with at least possibility $\lambda$, which is a predetermined confidence level for the profit of the manufacturer and the retailer in model (4).

Similar to [17], it is clear that model (4) can be transformed into model (5) in which the manufacturer and the retailer try to maximize their optimal -optimistic profits $\left[\bar{\Pi}_{m_{1}}\right]_{\lambda}^{U},\left[\bar{\Pi}_{m_{2}}\right]_{\lambda}^{U}$, and $\left[\bar{\Pi}_{r}\left(p_{1}, p_{2}\right)\right]_{\lambda}^{U}$. The other conditions satisfy that the marginal profit and consumer's demand are nonnegative. Model (4) can be transformed into the following, which is named optimistic manufacturer-leader Stackelberg (MSU) model.

$$
\begin{array}{ll}
\max _{w_{1}, t_{1}} & {\left[\bar{\Pi}_{m_{1}}\right]_{\lambda}^{U}=\left[\left(w_{1}-c_{1}\right) D_{1}-l_{1} t_{1}^{2}\right]_{\lambda}^{U}} \\
\max _{w_{2}, t_{2}} & {\left[\bar{\Pi}_{m_{2}}\right]_{\lambda}^{U}=\left[\left(w_{2}-c_{2}\right) D_{2}-l_{2} t_{2}^{2}\right]_{\lambda}^{U}} \\
\text { s.t. } & \text { Pos }\left\{w_{i}-c_{i}<0\right\}=0, \quad i=1,2, j=3-i . \\
& p_{1}^{*}, p_{2}^{*} \text { are derived from solving the problem } \\
& \begin{cases}\max _{p_{1}, p_{2}} & {\left[\bar{\Pi}_{r}\left(p_{1}, p_{2}\right)\right]_{\lambda}^{U}=\left[\sum_{i=1}^{2}\left(p_{i}-w_{i}\right) D_{i}\right]_{\lambda}^{U}} \\
\text { s.t. } & \operatorname{Pos}\left\{\alpha_{i}-\beta_{1} p_{i}+\beta_{2} p_{j}+\gamma_{1} t_{i}-\gamma_{2} t_{j}<0\right\}=0,\end{cases}
\end{array}
$$

Proposition 1. In the MSU model, given the wholesale prices and warranty levels made earlier by manufacturers, the retailer's optimal response functions, denoted by $p_{1}^{*}\left(w_{1}, t_{1}, t_{2}\right)$ and $p_{2}^{*}\left(w_{2}, t_{1}, t_{2}\right)$, are

$$
\begin{aligned}
& p_{1}^{*}\left(w_{1}, t_{1}, t_{2}\right)=\frac{1}{2} w_{1} \\
& +\frac{1}{2\left(\left(\beta_{1 \lambda}^{L}\right)^{2}-\left(\beta_{2 \lambda}^{U}\right)^{2}\right)}\left\{\left(\beta_{1 \lambda}^{L} \gamma_{1 \lambda}^{U}-\beta_{2 \lambda}^{U} \gamma_{2 \lambda}^{L}\right) t_{1}\right. \\
& \left.\quad+\left(\beta_{2 \lambda}^{U} \gamma_{1 \lambda}^{U}-\beta_{1 \lambda}^{L} \gamma_{2 \lambda}^{L}\right) t_{2}+\left(\beta_{1 \lambda}^{L} \alpha_{1 \lambda}^{U}+\beta_{2 \lambda}^{U} \alpha_{2 \lambda}^{U}\right)\right\}, \\
& p_{2}^{*}\left(w_{2}, t_{1}, t_{2}\right)=\frac{1}{2} w_{2} \\
& +\frac{1}{2\left(\left(\beta_{1 \lambda}^{L}\right)^{2}-\left(\beta_{2 \lambda}^{U}\right)^{2}\right)}\left\{\left(\beta_{2 \lambda}^{U} \gamma_{1 \lambda}^{U}-\beta_{1 \lambda}^{L} \gamma_{2 \lambda}^{L}\right) t_{1}\right. \\
& \left.\quad+\left(\beta_{1 \lambda}^{L} \gamma_{1 \lambda}^{U}-\beta_{2 \lambda}^{U} \gamma_{2 \lambda}^{L}\right) t_{2}+\left(\beta_{1 \lambda}^{L} \alpha_{2 \lambda}^{U}+\beta_{2 \lambda}^{U} \alpha_{1 \lambda}^{U}\right)\right\}
\end{aligned}
$$

under the conditions that $\operatorname{Pos}\left\{\alpha_{1}-\beta_{1} p_{1}^{*}\left(w_{1}, t_{1}, t_{2}\right)+\beta_{2} p_{2}^{*}\left(w_{2}\right.\right.$, $\left.\left.t_{1}, t_{2}\right)+\gamma_{1} t_{1}-\gamma_{2} t_{2}<0\right\}=0$ and $\operatorname{Pos}\left\{\alpha_{2}-\beta_{1} p_{2}^{*}\left(w_{2}, t_{1}, t_{2}\right)+\right.$ $\left.\beta_{2} p_{1}^{*}\left(w_{1}, t_{1}, t_{2}\right)+\gamma_{1} t_{2}-\gamma_{2} t_{1}<0\right\}=0$ hold.

Proof. The $\lambda$-optimistic value of the retailer's profit is

$$
\begin{aligned}
& {\left[\Pi_{r}\left(p_{1}, p_{2}\right)\right]_{\lambda}^{U}} \\
& =\left(p_{1}-w_{1}\right)\left(\alpha_{1 \lambda}^{U}-\beta_{1 \lambda}^{L} p_{1}+\beta_{2 \lambda}^{U} p_{2}+\gamma_{1 \lambda}^{U} t_{1}-\gamma_{2 \lambda}^{L} t_{2}\right) \\
& \quad+\left(p_{2}-w_{2}\right)\left(\alpha_{2 \lambda}^{U}-\beta_{1 \lambda}^{L} p_{2}+\beta_{2 \lambda}^{U} p_{1}+\gamma_{1 \lambda}^{U} t_{2}-\gamma_{2 \lambda}^{L} t_{1}\right) .
\end{aligned}
$$

The following first and second-order derivatives of $\left[\Pi_{r}\left(p_{1}, p_{2}\right)\right]_{\lambda}^{U}$ with respect to $\left(p_{1}, p_{2}\right)$ can be obtained:

$$
\begin{aligned}
\frac{\partial\left[\Pi_{r}\left(p_{1}, p_{2}\right)\right]_{\lambda}^{U}}{\partial p_{1}}= & \alpha_{1 \lambda}^{U}-2 \beta_{1 \lambda}^{L} p_{1}+2 \beta_{2 \lambda}^{U} p_{2}+\gamma_{1 \lambda}^{U} t_{1} \\
& -\gamma_{2 \lambda}^{L} t_{2}+\beta_{1 \lambda}^{L} w_{1}-\beta_{2 \lambda}^{U} w_{2}, \\
\frac{\partial\left[\Pi_{r}\left(p_{1}, p_{2}\right)\right]_{\lambda}^{U}}{\partial p_{2}}= & \alpha_{2 \lambda}^{U}-2 \beta_{1 \lambda}^{L} p_{2}+2 \beta_{2 \lambda}^{U} p_{1}+\gamma_{1 \lambda}^{U} t_{2} \\
\frac{\partial^{2}\left[\Pi_{r}\left(p_{1}, p_{2}\right)\right]_{\lambda}^{U}}{\partial p_{1}^{2}}= & \frac{\partial^{2}\left[\Pi_{r}\left(p_{1}, p_{2}\right)\right]_{\lambda}^{U}}{\partial p_{2}^{2}}=-2 \beta_{1 \lambda}^{L}, \\
\frac{\partial^{2}\left[\Pi_{r}\left(p_{1}, p_{2}\right)\right]_{\lambda}^{U}}{\partial p_{1} \partial p_{2}}= & \frac{\partial^{2}\left[\Pi_{r}\left(p_{1}, p_{2}\right)\right]_{\lambda}^{U}}{\partial p_{2} \partial p_{1}}=2 \beta_{2 \lambda}^{U} w_{1},
\end{aligned}
$$

Using the assumptions $\left(\beta_{1 \lambda}^{L}\right)^{2}-\left(\beta_{2 \lambda}^{U}\right)^{2}>0$ (since $E\left[\beta_{1}\right]>$ $\left.E\left[\beta_{2}\right]\right)$, the Hessian matrix is negative definite. Therefore, $\left[\Pi_{r}\left(p_{1}, p_{2}\right)\right]_{\lambda}^{U}$ is a concave function. Letting (8) and (9) be equal to zero, we can obtain (6).

Knowing the information of the retailer's reaction, the two manufacturers would use them to maximize their $\lambda$ optimistic profits by choosing the best wholesale prices and warranty levels, respectively.

Proposition 2. In the MSU model, the manufacturers' optimal wholesale prices (denoted by $w_{1}^{U *}$ and $w_{2}^{U *}$ ) and warranty levels (denoted by $t_{1}^{U *}$ and $t_{2}^{U *}$ ) are

$$
\begin{aligned}
& t_{1}^{U *}=\frac{A_{12} A_{23}-A_{22} A_{13}}{A_{11} A_{22}-A_{12} A_{21}}, \\
& t_{2}^{U *}=\frac{A_{21} A_{13}-A_{11} A_{23}}{A_{11} A_{22}-A_{12} A_{21}}, \\
& w_{1}^{U *}=\frac{4 l_{1 \lambda}^{L}}{\gamma_{1 \lambda}^{U}} t_{1}^{*}+c_{1 \lambda}^{L}, \\
& w_{2}^{U *}=\frac{4 l_{2 \lambda}^{L}}{\gamma_{1 \lambda}^{U}} t_{2}^{*}+c_{2 \lambda}^{L},
\end{aligned}
$$

where

$$
\begin{aligned}
& A_{11}=\gamma_{1 \lambda}^{U}-\frac{8 \beta_{1 \lambda}^{L} l_{1 \lambda}^{L}}{\gamma_{1 \lambda}^{U}}, \\
& A_{12}=\frac{4 \beta_{2 \lambda}^{U} l_{2 \lambda}^{L}}{\gamma_{1 \lambda}^{U}}-\gamma_{2 \lambda}^{L}, \\
& A_{13}=\alpha_{1 \lambda}^{U}-\beta_{1 \lambda}^{L} c_{1 \lambda}^{L}+\beta_{2 \lambda}^{U} c_{2 \lambda}^{L}, \\
& A_{21}=\frac{4 \beta_{2 \lambda}^{U} l_{1 \lambda}^{L}}{\gamma_{1 \lambda}^{U}}-\gamma_{2 \lambda}^{L},
\end{aligned}
$$




$$
\begin{aligned}
& A_{22}=\gamma_{1 \lambda}^{U}-\frac{8 \beta_{1 \lambda}^{L} l_{2 \lambda}^{L}}{\gamma_{1 \lambda}^{U}}, \\
& A_{23}=\alpha_{2 \lambda}^{U}-\beta_{1 \lambda}^{L} c_{2 \lambda}^{L}+\beta_{2 \lambda}^{U} c_{1 \lambda}^{L},
\end{aligned}
$$

under the conditions that $\operatorname{Pos}\left\{c_{1}>w_{1}^{U *}\right\}=0, \operatorname{Pos}\left\{c_{2}>w_{2}^{U *}\right\}=$ $0, \operatorname{Pos}\left\{\alpha_{1}-\beta_{1} p_{1}^{*}\left(w_{1}^{U *}, t_{1}^{U *}, t_{2}^{U *}\right)+\beta_{2} p_{2}^{*}\left(w_{2}^{U *}, t_{1}^{U *}, t_{2}^{U *}\right)+\gamma_{1} t_{1}^{U *}-\right.$ $\left.\gamma_{2} t_{2}^{U *}<0\right\}=0$, and $\operatorname{Pos}\left\{\alpha_{2}-\beta_{1} p_{2}^{*}\left(w_{2}^{U *}, t_{1}^{U *}, t_{2}^{U *}\right)+\right.$ $\left.\beta_{2} p_{1}^{*}\left(w_{1}^{U *}, t_{1}^{U *}, t_{2}^{U *}\right)+\gamma_{1} t_{2}^{U *}-\gamma_{2} t_{1}^{U *}<0\right\}=0$ hold.

Proof. Substituting (6) into (3) results in

$$
\begin{aligned}
& {\left[\Pi_{m_{i}}\right]_{\lambda}^{U}=\left(w_{i}-c_{i \lambda}^{L}\right)} \\
& \quad \cdot\left(-\frac{\beta_{1 \lambda}^{L} w_{i}}{2}+\frac{\beta_{2 \lambda}^{U} w_{j}}{2}+\frac{\gamma_{1 \lambda}^{U} t_{i}}{2}-\frac{\gamma_{2 \lambda}^{L} t_{j}}{2}+\frac{\alpha_{i \lambda}^{U}}{2}\right) \\
& \quad-l_{i \lambda}^{L} t_{i}^{2}, \quad i=1,2, \quad j=3-i .
\end{aligned}
$$

The first- and second-order partial derivative with respect to $\left(w_{i}, t_{i}\right)$ can be shown as

$$
\begin{aligned}
\frac{\partial\left[\Pi_{m_{i}}\right]_{\lambda}^{U}}{\partial w_{i}}= & -\beta_{1 \lambda}^{L} w_{i}+\frac{\beta_{2 \lambda}^{U} w_{j}}{2}+\frac{\gamma_{1 \lambda}^{U} t_{i}}{2}-\frac{\gamma_{2 \lambda}^{L} t_{j}}{2}+\frac{\alpha_{i \lambda}^{U}}{2} \\
& +\frac{c_{i \lambda}^{L} \beta_{1 \lambda}^{L}}{2}, \\
\frac{\partial\left[\Pi_{m_{i}}\right]_{\lambda}^{U}}{\partial t_{i}}= & \frac{\gamma_{1 \lambda}^{U}}{2}\left(w_{i}-c_{i \lambda}^{L}\right)-2 l_{i \lambda}^{L} t_{i} . \\
\frac{\partial^{2}\left[\Pi_{m_{i}}\right]_{\lambda}^{U}}{\partial w_{i}^{2}}= & -\beta_{1 \lambda}^{L}<0,
\end{aligned}
$$

$$
\begin{aligned}
& \frac{\partial^{2}\left[\Pi_{m_{i}}\right]_{\lambda}^{U}}{\partial t_{i}^{2}}=-2 l_{i \lambda}^{L}, \\
& \frac{\partial^{2}\left[\Pi_{m_{i}}\right]_{\lambda}^{U}}{\partial w_{i} \partial t_{i}}=\frac{\partial^{2}\left[\Pi_{m_{i}}\right]_{\lambda}^{U}}{\partial t_{i} \partial w_{i}}=\frac{\gamma_{1 \lambda}^{U}}{2} .
\end{aligned}
$$

Since $8 \beta_{1 \lambda}^{L} l_{i \lambda}^{L}-\left(\gamma_{1 \lambda}^{U}\right)^{2}>0,\left[\Pi_{m_{i}}\right]_{\lambda}^{U}$ are concave functions. Setting (15) and (16) to be equal to zero and solving them, we can attain (12).

Substituting (12) into (6), then the retailer's optimal retail prices are

$$
\begin{aligned}
p_{1}^{U *} & =\frac{1}{2} w_{1}^{U *} \\
& +\frac{1}{2\left(\left(\beta_{1 \lambda}^{L}\right)^{2}-\left(\beta_{2 \lambda}^{U}\right)^{2}\right)}\left\{\left(\beta_{1 \lambda}^{L} \gamma_{1 \lambda}^{U}-\beta_{2 \lambda}^{U} \gamma_{2 \lambda}^{L}\right) t_{1}^{U *}\right. \\
& \left.+\left(\beta_{2 \lambda}^{U} \gamma_{1 \lambda}^{U}-\beta_{1 \lambda}^{L} \gamma_{2 \lambda}^{L}\right) t_{2}^{U *}+\left(\beta_{1 \lambda}^{L} \alpha_{1 \lambda}^{U}+\beta_{2 \lambda}^{U} \alpha_{2 \lambda}^{U}\right)\right\}, \\
p_{2}^{U *} & =\frac{1}{2} w_{2}^{U *} \\
& +\frac{1}{2\left(\left(\beta_{1 \lambda}^{L}\right)^{2}-\left(\beta_{2 \lambda}^{U}\right)^{2}\right)}\left\{\left(\beta_{2 \lambda}^{U} \gamma_{1 \lambda}^{U}-\beta_{1 \lambda}^{L} \gamma_{2 \lambda}^{L}\right) t_{1}^{U *}\right. \\
& \left.+\left(\beta_{1 \lambda}^{L} \gamma_{1 \lambda}^{U}-\beta_{2 \lambda}^{U} \gamma_{2 \lambda}^{L}\right) t_{2}^{U *}+\left(\beta_{1 \lambda}^{L} \alpha_{2 \lambda}^{U}+\beta_{2 \lambda}^{U} \alpha_{1 \lambda}^{U}\right)\right\} .
\end{aligned}
$$

Therefore, we can get a unique $\lambda$-optimistic Stackelberg equilibrium $w_{i}^{U *}, t_{i}^{U *}$, and $p_{i}^{U *}(i=1,2)$ of model (4).

3.2. The $\lambda$-Pessimistic Manufacturer-Leader Stackelberg (MSL) Model. The minimax chance-constrained programming model for the manufacturer-leader Stackelberg supply chain can be formulated as follows, where $\lambda$ is a predetermined confidence level for the channel members' profits.

$$
\begin{array}{ll}
\max _{w_{1}, t_{1}} & \min _{\bar{\Pi}_{m_{1}}} \bar{\Pi}_{m_{1}}\left(w_{1}\right) \\
\max _{w_{2}, t_{2}} & \min _{\bar{\Pi}_{m_{2}}} \bar{\Pi}_{m_{2}}\left(w_{2}\right) \\
\text { s.t. } & \operatorname{Pos}\left\{\left(w_{i}-c_{i}\right)\left(\alpha_{i}-\beta_{1} p_{i}^{*}\left(w_{i}, t_{i}, t_{j}\right)+\beta_{2} p_{j}^{*}\left(w_{j}, t_{j}, t_{i}\right)+\gamma_{1} t_{i}-\gamma_{2} t_{j}\right) \leq \bar{\Pi}_{m_{i}}\right\} \geq \lambda, \\
& \operatorname{Pos}\left\{w_{i}-c_{i}<0\right\}=0, \quad i=1,2, j=3-i . \\
& p_{1}^{*}, p_{2}^{*} \text { are derived from solving the problem } \\
& \begin{cases}\max _{p_{1}, p_{2}} \quad \min \bar{\Pi}_{r}\left(p_{1}, p_{2}\right) \\
\operatorname{s.t.} \quad & \operatorname{Pos}\left\{\left(p_{i}-w_{i}\right)\left(\alpha_{i}-\beta_{1} p_{i}+\beta_{2} p_{j}+\gamma_{1} t_{i}-\gamma_{2} t_{j}\right) \leq \bar{\Pi}_{r}\right\} \geq \lambda, \\
& \operatorname{Pos}\left\{\alpha_{i}-\beta_{1} p_{i}+\beta_{2} p_{j}+\gamma_{1} t_{i}-\gamma_{2} t_{j}<0\right\}=0, \\
& p_{i} \geq w_{i}, \quad i=1,2, j=3-i .\end{cases}
\end{array}
$$


When the two manufacturers and retailer's profits function to achieve with at least possibility $\lambda, \bar{\Pi}_{m_{i}}$ and $\bar{\Pi}_{r}$ should be the minimum value. The channel members try to maximize their optimal $\lambda$-pessimistic profits and model (21) can be transformed into the following.

$$
\begin{array}{ll}
\max _{w_{1}, t_{1}} & {\left[\bar{\Pi}_{m_{1}}\right]_{\lambda}^{L}=\left[\left(w_{1}-c_{1}\right) D_{1}-l_{1} t_{1}^{2}\right]_{\lambda}^{L}} \\
\max _{w_{2}, t_{2}} & {\left[\bar{\Pi}_{m_{2}}\right]_{\lambda}^{L}=\left[\left(w_{2}-c_{2}\right) D_{2}-l_{2} t_{2}^{2}\right]_{\lambda}^{L}} \\
\text { s.t. } & \text { Pos }\left\{w_{i}-c_{i}<0\right\}=0, \quad i=1,2, \quad j=3-i . \\
& p_{1}^{*}, p_{2}^{*} \text { are derived from solving the problem } \\
& \begin{cases}\max _{p_{1}, p_{2}} & {\left[\bar{\Pi}_{r}\left(p_{1}, p_{2}\right)\right]_{\lambda}^{L}=\left[\sum_{i=1}^{2}\left(p_{i}-w_{i}\right) D_{i}\right]_{\lambda}^{L}} \\
\text { s.t. } & \operatorname{Pos}\left\{\alpha_{i}-\beta_{1} p_{i}+\beta_{2} p_{j}+\gamma_{1} t_{i}-\gamma_{2} t_{j}<0\right\}=0,\end{cases}
\end{array}
$$

Similar to the proof of Section 3.1, we can easily obtain Proposition 3 as follows.

Proposition 3. In the MSL model, the unique $\lambda$-pessimistic Stackelberg equilibrium solutions are

$$
\begin{aligned}
t_{1}^{L *} & =\frac{B_{12} B_{23}-B_{22} B_{13}}{B_{11} B_{22}-B_{12} B_{21}}, \\
t_{2}^{L *} & =\frac{B_{21} B_{13}-B_{11} B_{23}}{B_{11} B_{22}-B_{12} B_{21}}, \\
w_{1}^{L *} & =\frac{4 l_{1 \lambda}^{U}}{\gamma_{1 \lambda}^{L}} t_{1}^{*}+c_{1 \lambda}^{U}, \\
w_{2}^{L *} & =\frac{4 l_{2 \lambda}^{U}}{\gamma_{1 \lambda}^{L}} t_{2}^{*}+c_{2 \lambda}^{U}, \\
p_{1}^{L *} & =\frac{1}{2} w_{1}^{L *} \\
& +\frac{1}{2\left(\left(\beta_{1 \lambda}^{U}\right)^{2}-\left(\beta_{2 \lambda}^{L}\right)^{2}\right)}\left\{\left(\beta_{1 \lambda}^{U} \gamma_{1 \lambda}^{L}-\beta_{2 \lambda}^{L} \gamma_{2 \lambda}^{U}\right) t_{1}^{L *}\right. \\
& \left.+\left(\beta_{2 \lambda}^{L} \gamma_{1 \lambda}^{L}-\beta_{1 \lambda}^{U} \gamma_{2 \lambda}^{U}\right) t_{2}^{L *}+\left(\beta_{1 \lambda}^{U} \alpha_{1 \lambda}^{L}+\beta_{2 \lambda}^{L} \alpha_{2 \lambda}^{L}\right)\right\}, \\
p_{2}^{L *} & =\frac{1}{2} w_{2}^{L *} \\
& +\frac{1}{2\left(\left(\beta_{1 \lambda}^{U}\right)^{2}-\left(\beta_{2 \lambda}^{L}\right)^{2}\right)}\left\{\left(\beta_{2 \lambda}^{L} \gamma_{1 \lambda}^{L}-\beta_{1 \lambda}^{U} \gamma_{2 \lambda}^{U}\right) t_{1}^{L *}\right. \\
& \left.+\left(\beta_{1 \lambda}^{U} \gamma_{1 \lambda}^{L}-\beta_{2 \lambda}^{L} \gamma_{2 \lambda}^{U}\right) t_{2}^{L *}+\left(\beta_{1 \lambda}^{U} \alpha_{2 \lambda}^{L}+\beta_{2 \lambda}^{L} \alpha_{1 \lambda}^{L}\right)\right\},
\end{aligned}
$$

where

$$
\begin{aligned}
& B_{11}=\gamma_{1 \lambda}^{L}-\frac{8 \beta_{1 \lambda}^{U} l_{1 \lambda}^{U}}{\gamma_{1 \lambda}^{L}}, \\
& B_{12}=\frac{4 \beta_{2 \lambda}^{L} l_{2 \lambda}^{U}}{\gamma_{1 \lambda}^{L}}-\gamma_{2 \lambda}^{U},
\end{aligned}
$$

$$
\begin{aligned}
& B_{13}=\alpha_{1 \lambda}^{L}-\beta_{1 \lambda}^{U} c_{1 \lambda}^{U}+\beta_{2 \lambda}^{L} c_{2 \lambda}^{U}, \\
& B_{21}=\frac{4 \beta_{2 \lambda}^{L} l_{1 \lambda}^{U}}{\gamma_{1 \lambda}^{L}}-\gamma_{2 \lambda}^{U}, \\
& B_{22}=\gamma_{1 \lambda}^{L}-\frac{8 \beta_{1 \lambda}^{U} l_{2 \lambda}^{U}}{\gamma_{1 \lambda}^{L}}, \\
& B_{23}=\alpha_{2 \lambda}^{L}-\beta_{1 \lambda}^{U} c_{2 \lambda}^{U}+\beta_{2 \lambda}^{L} c_{1 \lambda}^{U},
\end{aligned}
$$

under the conditions that $\operatorname{Pos}\left\{c_{1}>w_{1}^{L *}\right\}=0$, Pos $\left\{c_{2}>w_{2}^{L *}\right\}=$ $0, \operatorname{Pos}\left\{\alpha_{1}-\beta_{1} p_{1}^{*}\left(w_{1}^{L *}, t_{1}^{L *}, t_{2}^{L *}\right)+\beta_{2} p_{2}^{*}\left(w_{2}^{L *}, t_{1}^{L *}, t_{2}^{L *}\right)+\gamma_{1} t_{1}^{L *}-\right.$ $\left.\gamma_{2} t_{2}^{L *}<0\right\}=0$, and $\operatorname{Pos}\left\{\alpha_{2}-\beta_{1} p_{2}^{*}\left(w_{2}^{L *}, t_{1}^{L *}, t_{2}^{L *}\right)+\right.$ $\left.\beta_{2} p_{1}^{*}\left(w_{1}^{L *}, t_{1}^{L *}, t_{2}^{L *}\right)+\gamma_{1} t_{2}^{L *}-\gamma_{2} t_{1}^{L *}<0\right\}=0$ hold.

3.3. The Expected Value Manufacturer-Leader Stackelberg (MSE) Model. In this subsection, we formulate the following manufacturer-leader Stackelberg expected value model.

$$
\begin{array}{ll}
\max _{w_{1}, t_{1}} & E\left[\Pi_{m_{1}}\right]=E\left[\left(w_{1}-c_{1}\right) D_{1}-l_{1} t_{1}^{2}\right] \\
\max _{w_{2}, t_{2}} & E\left[\Pi_{m_{2}}\right]=E\left[\left(w_{2}-c_{2}\right) D_{1}-l_{2} t_{2}^{2}\right] \\
\text { s.t. } & \operatorname{Pos}\left\{w_{i}-c_{i}<0\right\}=0, \quad i=1,2, j=3-i . \\
& p_{1}^{*}, p_{2}^{*} \text { are derived from solving the problem } \\
& \begin{cases}\max _{p_{1}, p_{2}} & E\left[\Pi_{r}\left(p_{1}, p_{2}\right)\right]=E\left[\sum_{i=1}^{2}\left(p_{i}-w_{i}\right) D_{i}\right] \\
\text { s.t. } & \operatorname{Pos}\left\{\alpha_{i}-\beta_{1} p_{i}+\beta_{2} p_{j}+\gamma_{1} t_{i}-\gamma_{2} t_{j}<0\right\}=0,\end{cases}
\end{array}
$$

Similar to the proof of Section 3.1, we can get the following Proposition 4.

Proposition 4. The unique expected Stackelberg equilibrium solutions of the MSE model (25) are

$$
\begin{aligned}
t_{1}^{E *} & =\frac{F_{12} F_{23}-F_{22} F_{13}}{F_{11} F_{22}-F_{12} F_{21}}, \\
t_{2}^{E *} & =\frac{F_{21} F_{13}-F_{11} F_{23}}{F_{11} F_{22}-F_{12} F_{21}}, \\
w_{1}^{E *} & =\frac{4 E\left[l_{1}\right]}{E\left[\gamma_{1}\right]} t_{1}^{E *}+F_{10}, \\
w_{2}^{E *} & =\frac{4 E\left[l_{2}\right]}{E\left[\gamma_{1}\right]} t_{2}^{E *}+F_{20}, \\
p_{1}^{E *} & =\frac{1}{2} w_{1}^{E *}+\frac{1}{2\left(E\left[\beta_{1}\right]^{2}-E\left[\beta_{2}\right]^{2}\right)}\left\{\left(E\left[\beta_{1}\right] E\left[\gamma_{1}\right]\right.\right. \\
& \left.-E\left[\beta_{2}\right] E\left[\gamma_{2}\right]\right) t_{1}^{E *}+\left(E\left[\beta_{2}\right] E\left[\gamma_{1}\right]\right. \\
& \left.-E\left[\beta_{1}\right] E\left[\gamma_{2}\right]\right) t_{2}^{E *}+\left(E\left[\beta_{1}\right] E\left[\alpha_{1}\right]\right. \\
& \left.\left.+E\left[\beta_{2}\right] E\left[\alpha_{2}\right]\right)\right\},
\end{aligned}
$$




$$
\begin{aligned}
p_{2}^{E *} & =\frac{1}{2} w_{2}+\frac{1}{2\left(E\left[\beta_{1}\right]^{2}-E\left[\beta_{2}\right]^{2}\right)}\left\{\left(E\left[\beta_{2}\right] E\left[\gamma_{1}\right]\right.\right. \\
& \left.-E\left[\beta_{1}\right] E\left[\gamma_{2}\right]\right) t_{1}^{E *}+\left(E\left[\beta_{1}\right] E\left[\gamma_{1}\right]\right. \\
& \left.-E\left[\beta_{2}\right] E\left[\gamma_{2}\right]\right) t_{2}^{E *}+\left(E\left[\beta_{1}\right] E\left[\alpha_{2}\right]\right. \\
& \left.\left.+E\left[\beta_{2}\right] E\left[\alpha_{1}\right]\right)\right\}
\end{aligned}
$$

where

$$
\begin{aligned}
& F_{10}=\frac{1}{E\left[\gamma_{1}\right]}\left\{-2 E\left[c_{1} \beta_{1}\right]\right. \\
& \cdot \frac{E\left[\beta_{1}\right] E\left[\gamma_{1}\right]-E\left[\beta_{2}\right] E\left[\gamma_{2}\right]}{2\left(E\left[\beta_{1}\right]^{2}-E\left[\beta_{2}\right]^{2}\right)} \\
& +\int_{0}^{1}\left(c_{1 \lambda}^{L} \beta_{2 \lambda}^{U}+c_{1 \lambda}^{U} \beta_{2 \lambda}^{L}\right) d \lambda \\
& \cdot \frac{E\left[\beta_{2}\right] E\left[\gamma_{1}\right]-E\left[\beta_{1}\right] E\left[\gamma_{2}\right]}{2\left(E\left[\beta_{1}\right]^{2}-E\left[\beta_{2}\right]^{2}\right)} \\
& \left.+\int_{0}^{1}\left(c_{1 \lambda}^{L} \gamma_{1 \lambda}^{U}+c_{1 \lambda}^{U} \gamma_{1 \lambda}^{L}\right) d \lambda\right\} \text {, } \\
& F_{20}=\frac{1}{E\left[\gamma_{1}\right]}\left\{-2 E\left[c_{2} \beta_{1}\right]\right. \\
& \frac{E\left[\beta_{1}\right] E\left[\gamma_{1}\right]-E\left[\beta_{2}\right] E\left[\gamma_{2}\right]}{2\left(E\left[\beta_{1}\right]^{2}-E\left[\beta_{2}\right]^{2}\right)} \\
& +\int_{0}^{1}\left(c_{2 \lambda}^{L} \beta_{2 \lambda}^{U}+c_{2 \lambda}^{U} \beta_{2 \lambda}^{L}\right) d \lambda \\
& \cdot \frac{E\left[\beta_{2}\right] E\left[\gamma_{1}\right]-E\left[\beta_{1}\right] E\left[\gamma_{2}\right]}{2\left(E\left[\beta_{1}\right]^{2}-E\left[\beta_{2}\right]^{2}\right)} \\
& \left.+\int_{0}^{1}\left(c_{2 \lambda}^{L} \gamma_{1 \lambda}^{U}+c_{2 \lambda}^{U} \gamma_{2 \lambda}^{L}\right) d \lambda\right\}, \\
& F_{11}=E\left[\gamma_{1}\right]-\frac{8 E\left[\beta_{1}\right] E\left[l_{1}\right]}{E\left[\gamma_{1}\right]}, \\
& F_{12}=\frac{4 E\left[\beta_{2}\right] E\left[l_{2}\right]}{E\left[\gamma_{1}\right]}-E\left[\gamma_{2}\right] \text {, } \\
& F_{21}=\frac{4 E\left[\beta_{2}\right] E\left[l_{1}\right]}{E\left[\gamma_{1}\right]}-E\left[\gamma_{2}\right], \\
& F_{22}=E\left[\gamma_{1}\right]-\frac{8 E\left[\beta_{1}\right] E\left[l_{2}\right]}{E\left[\gamma_{1}\right]},
\end{aligned}
$$

$$
\begin{aligned}
& F_{13}=E\left[\alpha_{1}\right]-2 E\left[\beta_{1}\right] F_{10}+E\left[\beta_{2}\right] F_{20}+E\left[c_{1} \beta_{1}\right], \\
& F_{23}=E\left[\alpha_{2}\right]+E\left[\beta_{2}\right] F_{10}-2 E\left[\beta_{1}\right] F_{20}+E\left[c_{2} \beta_{1}\right] .
\end{aligned}
$$

Remark 5. If two manufacturers' warranty services are not considered, the optimal pricing decisions, where the manufacturers and the retailer try to maximize their $\lambda$-optimistic profits, can be chosen as

$$
\begin{aligned}
& w_{1}^{U 0 *} \\
& =\frac{\left(2 \beta_{1 \lambda}^{L} \alpha_{1 \lambda}^{U}+\beta_{2 \lambda}^{U} \alpha_{2 \lambda}^{U}\right)+\left(2 \beta_{1 \lambda}^{L} \beta_{1 \lambda}^{L} c_{1 \lambda}^{L}+\beta_{1 \lambda}^{L} \beta_{2 \lambda}^{U} c_{2 \lambda}^{L}\right)}{4\left(\beta_{1 \lambda}^{L}\right)^{2}-\left(\beta_{2 \lambda}^{U}\right)^{2}}, \\
& w_{2}^{U 0 *} \\
& =\frac{\left(2 \beta_{1 \lambda}^{L} \alpha_{2 \lambda}^{U}+\beta_{2 \lambda}^{U} \alpha_{1 \lambda}^{U}\right)+\left(2 \beta_{1 \lambda}^{L} \beta_{1 \lambda}^{L} c_{2 \lambda}^{L}+\beta_{1 \lambda}^{L} \beta_{2 \lambda}^{U} c_{1 \lambda}^{L}\right)}{4\left(\beta_{1 \lambda}^{L}\right)^{2}-\left(\beta_{2 \lambda}^{U}\right)^{2}}, \\
& p_{1}^{U 0 *}=\frac{1}{2} w_{1}^{U 0 *}+\frac{\beta_{1 \lambda}^{U} \alpha_{1 \lambda}^{L}+\beta_{2 \lambda}^{L} \alpha_{2 \lambda}^{L}}{2\left(\beta_{1 \lambda}^{U}\right)^{2}-2\left(\beta_{2 \lambda}^{L}\right)^{2}}, \\
& p_{2}^{U 0 *}=\frac{1}{2} w_{2}^{U 0 *}+\frac{\beta_{1 \lambda}^{U} \alpha_{2 \lambda}^{L}+\beta_{2 \lambda}^{L} \alpha_{1 \lambda}^{L}}{2\left(\beta_{1 \lambda}^{U}\right)^{2}-2\left(\beta_{2 \lambda}^{L}\right)^{2}} .
\end{aligned}
$$

Remark 6. Considering only one manufacturer's warranty level, without loss of generality, we assume that $m_{1}$ provides the warranty for the retailer, and the unique $\lambda$-optimistic Stackelberg equilibrium solutions of this model are as follows:

$$
\begin{aligned}
w_{1}^{U 1 *}= & \frac{2 \beta_{1 \lambda}^{L} G_{12}+\beta_{2 \lambda}^{U} G_{22}}{2 \beta_{1 \lambda}^{L} G_{11}+\beta_{2 \lambda}^{U} G_{21}}, \\
w_{2}^{U 1 *}= & \frac{G_{11} G_{22}-G_{12} G_{21}}{2 \beta_{1 \lambda}^{L} G_{11}+\beta_{2 \lambda}^{U} G_{21}}, \\
t_{1}^{U 1 *}= & \frac{\gamma_{1 \lambda}^{U} w_{1}^{U 1 *}}{4 l_{1 \lambda}^{L}}-\frac{\gamma_{1 \lambda}^{U} c_{1 \lambda}^{L}}{4 l_{1 \lambda}^{L}}, \\
p_{1}^{U 1 *}= & \frac{1}{2} w_{1}^{U 1 *}+\frac{\beta_{1 \lambda}^{L} \gamma_{1 \lambda}^{U}-\beta_{2 \lambda}^{U} \gamma_{1 \lambda}^{L}}{2\left(\beta_{1 \lambda}^{U}\right)^{2}-2\left(\beta_{2 \lambda}^{L}\right)^{2}} t_{1}^{U 1 *} \\
& +\frac{\beta_{1 \lambda}^{U} \alpha_{1 \lambda}^{L}+\beta_{2 \lambda}^{L} \alpha_{2 \lambda}^{L}}{2\left(\beta_{1 \lambda}^{U}\right)^{2}-2\left(\beta_{2 \lambda}^{L}\right)^{2}}, \\
p_{2}^{U 1 *}= & \frac{1}{2} w_{2}^{U 1 *}+\frac{\beta_{2 \lambda}^{U} \gamma_{1 \lambda}^{U}-\beta_{1 \lambda}^{L} \gamma_{1 \lambda}^{L}}{2\left(\beta_{1 \lambda}^{U}\right)^{2}-2\left(\beta_{2 \lambda}^{L}\right)^{2}} t_{2}^{U 1 *} \\
& +\frac{\beta_{1 \lambda}^{U} \alpha_{2 \lambda}^{L}+\beta_{2 \lambda}^{L} \alpha_{1 \lambda}^{L}}{2\left(\beta_{1 \lambda}^{U}\right)^{2}-2\left(\beta_{2 \lambda}^{L}\right)^{2}},
\end{aligned}
$$

where

$$
G_{11}=2 \beta_{1 \lambda}^{L}-\frac{\gamma_{1 \lambda}^{U} \gamma_{1 \lambda}^{U}}{4 l_{1 \lambda}^{L}}
$$


TABLE 2: Relation between linguistic expression and triangular fuzzy variable.

\begin{tabular}{lcc}
\hline & Linguistic expression & Triangular fuzzy variable \\
\hline$c_{1}$ & Low (about 2) & $(1,2,3)$ \\
& High (about 6) & $(5,6,7)$ \\
$c_{2}$ & Low (about 6) & $(5,6,7)$ \\
& High (about 10) & $(8,10,12)$ \\
$\alpha_{1}$ & Small (about 1500) & $(1300,1500,1700)$ \\
& Large (about 2000) & $(1900,2000,2100)$ \\
$\alpha_{2}$ & Small (about 600) & $(400,600,800)$ \\
& Large (about 1000) & $(950,1000,1050)$ \\
$\beta_{1}$ & Sensitive (about 150) & $(120,150,180)$ \\
& Very sensitive (about 200) & $(180,200,220)$ \\
$\beta_{2}$ & Sensitive (about 60) & $(50,60,70)$ \\
& Very sensitive (about 100) & $(90,100,110)$ \\
$\gamma_{1}$ & Sensitive (about 20) & $(10,20,30)$ \\
& Very sensitive (about 50) & $(40,50,60)$ \\
$\gamma_{2}$ & Sensitive (about 10) & $(5,10,15)$ \\
& Very sensitive (about 20) & $(10,20,30)$ \\
$l_{1}$ & Sensitive (about 15) & $(12,15,18)$ \\
& Very sensitive (about 25) & $(20,25,30)$ \\
$l_{2}$ & Sensitive (about 10) & $(8,10,12)$ \\
& Very sensitive (about 15) & $(10,15,20)$ \\
\hline
\end{tabular}

$$
\begin{aligned}
& G_{21}=\frac{\gamma_{1 \lambda}^{U} \gamma_{1 \lambda}^{L}}{4 l_{1 \lambda}^{L}}-2 \beta_{2 \lambda}^{U}, \\
& G_{12}=\alpha_{1 \lambda}^{U}-\frac{c_{1 \lambda}^{L} \gamma_{1 \lambda}^{U} \gamma_{1 \lambda}^{U}}{4 l_{1 \lambda}^{L}}+c_{1 \lambda}^{L} \beta_{1 \lambda}^{L}, \\
& G_{22}=\alpha_{2 \lambda}^{U}+\frac{c_{1 \lambda}^{L} \gamma_{1 \lambda}^{U} \gamma_{1 \lambda}^{L}}{4 l_{1 \lambda}^{L}}+c_{2 \lambda}^{L} \beta_{1 \lambda}^{L} .
\end{aligned}
$$

\section{Numerical Example}

The pricing and warranty strategies for different Stackelberg, that is, the $\lambda$-optimistic, the $\lambda$-pessimistic, and the expected value Stackelberg, of the supply chain have been discussed. All the expressions are in a very complicated form; therefore, we use numerical examples to compare the optimal results in the different decision scenarios and to analyze the effect of some key parameters on the equilibrium solutions. Assume that the relation between linguistic expressions and triangular fuzzy variables for the manufacturing costs, primary market bases, price elastic coefficients, warranty elastic coefficients, and warranty cost coefficients are determined by experts' experiences as shown in Table 2. On the basis of comparison and analysis, some managerial insights are derived.

Consider the case that the manufacturing costs are in low levels, the primary market bases are in large levels, and all the elastic coefficients are in very sensitive levels. By using Table 2 , the expected values of all triangular fuzzy variables are
TABLE 3: The optimal decisions of prices and warranty levels.

\begin{tabular}{lcccccc}
\hline Scenario & $w_{1}^{*}$ & $w_{2}^{*}$ & $t_{1}^{*}$ & $t_{2}^{*}$ & $p_{1}^{*}$ & $p_{2}^{*}$ \\
\hline MSU & 8.467 & 7.755 & 3.611 & 1.815 & 13.735 & 11.474 \\
MSE & 8.244 & 7.602 & 3.208 & 1.478 & 12.908 & 10.718 \\
MSL & 7.999 & 7.410 & 2.676 & 0.908 & 12.146 & 10.008 \\
\hline
\end{tabular}

TABLE 4: The maximal profits in different models.

\begin{tabular}{lcccc}
\hline Scenario & $\Pi_{m_{1}}$ & $\Pi_{m_{2}}$ & $\Pi_{r}$ & $\Pi_{t}$ \\
\hline MSU & 4042.4 & 328.2 & 4154.5 & 8525.1 \\
MSE & 3621.0 & 218.5 & 3385.3 & 7224.8 \\
MSL & 3243.6 & 136.2 & 2773.5 & 6153.3 \\
\hline
\end{tabular}

$E\left[c_{1}\right]=(1+2 \times 2+3) / 4=2, E\left[c_{2}\right]=(5+2 \times 6+7) / 4=6, E\left[\alpha_{1}\right]=$ $2000, E\left[\alpha_{2}\right]=1000, E\left[\beta_{1}\right]=200, E\left[\beta_{2}\right]=100, E\left[\gamma_{1}\right]=$ $50, E\left[\gamma_{2}\right]=20, E\left[l_{1}\right]=25$, and $E\left[l_{2}\right]=15$. The $\lambda$-optimistic and $\lambda$-pessimistic values are, respectively, $c_{1 \lambda}^{U}=1+\lambda, c_{1 \lambda}^{L}=$ $3-\lambda, c_{2 \lambda}^{U}=5+\lambda, c_{2 \lambda}^{L}=7-\lambda, \alpha_{1 \lambda}^{U}=1900+100 \lambda$, $\alpha_{1 \lambda}^{L}=2100-100 \lambda, \alpha_{2 \lambda}^{U}=950+50 \lambda, \alpha_{2 \lambda}^{L}=1050-50 \lambda, \beta_{1 \lambda}^{U}=$ $180+20 \lambda, \beta_{1 \lambda}^{L}=220-20 \lambda, \beta_{2 \lambda}^{U}=90+10 \lambda, \beta_{2 \lambda}^{L}=110-10 \lambda$, $\gamma_{1 \lambda}^{U}=40+10 \lambda, \gamma_{1 \lambda}^{L}=60-10 \lambda, \gamma_{2 \lambda}^{U}=10+10 \lambda, \gamma_{2 \lambda}^{L}=30-10 \lambda$, $l_{1 \lambda}^{U}=20+5 \lambda, l_{1 \lambda}^{L}=30-5 \lambda, l_{2 \lambda}^{U}=10+5 \lambda$, and $l_{2 \lambda}^{L}=20-5 \lambda$. We can also calculate $E\left[c_{1} \beta_{1}\right]=406.67, E\left[c_{2} \beta_{1}\right]=1206.7$, $(1 / 2) \int_{0}^{1}\left(c_{1 \lambda}^{L} \beta_{2 \lambda}^{U}+c_{1 \lambda}^{U} \beta_{2 \lambda}^{L}\right) d \lambda=196.67,(1 / 2) \int_{0}^{1}\left(c_{1 \lambda}^{L} \gamma_{1 \lambda}^{U}+\right.$ $\left.c_{1 \lambda}^{U} \gamma_{1 \lambda}^{L}\right) d \lambda=96.67,(1 / 2) \int_{0}^{1}\left(c_{2 \lambda}^{L} \beta_{2 \lambda}^{U}+c_{2 \lambda}^{U} \beta_{2 \lambda}^{L}\right) d \lambda=596.67$, and $(1 / 2) \int_{0}^{1}\left(c_{2 \lambda}^{L} \gamma_{1 \lambda}^{U}+c_{2 \lambda}^{U} \gamma_{1 \lambda}^{L}\right) d \lambda=296.67$.

Discussion 1 (comparison of the optimal decisions in different models). From the results obtained in the above scenarios, the optimal wholesale prices, warranty levels, retail prices, and the maximal profits of each channel member can be calculated as shown in Tables 3 and 4 with a confidence value $\lambda=0.8$.

Tables 3 and 4 indicate that two substitutable products have different wholesale prices, different retail prices, and different warranty levels in the MSU, MSE, and MSL models. Therefore, the following insights are obtained.

(1-1) From Table 2, we can see that $w_{i}^{U *}>w_{i}^{E *}>w_{i}^{L *}$, $t_{i}^{U *}>t_{i}^{E *}>t_{i}^{L *}, p_{i}^{U *}>p_{i}^{E *}>p_{i}^{L *}, \Pi_{m_{i}}^{U *}>\Pi_{m_{i}}^{E *}>\Pi_{m_{i}}^{L *}$, $\Pi_{r}^{U *}>\Pi_{r}^{E *}>\Pi_{r}^{L *}$, and $\Pi_{t}^{U *}>\Pi_{t}^{E *}>\Pi_{t}^{L *}(i=1,2)$; that is, the firm's optimal pricing and warranty strategy and the corresponding profits vary with the ranking measure. The strategy in the $\lambda$-optimistic Stackelberg model is always the highest, and the strategy in the $\lambda$-pessimistic Stackelberg model is always the lowest.

(1-2) The total profits of the two manufacturers are higher than that of the retailer; that is because the manufacturers play the leader's role which hold advantage in acquiring more profits in this paper.

Discussion 2 (the influence of the manufacturers' warranty levels on equilibrium solutions). Considering three different scenarios (a) there is no warranty service to be considered in the supply chain, (b) just manufacturer $m_{1}$ provides the 

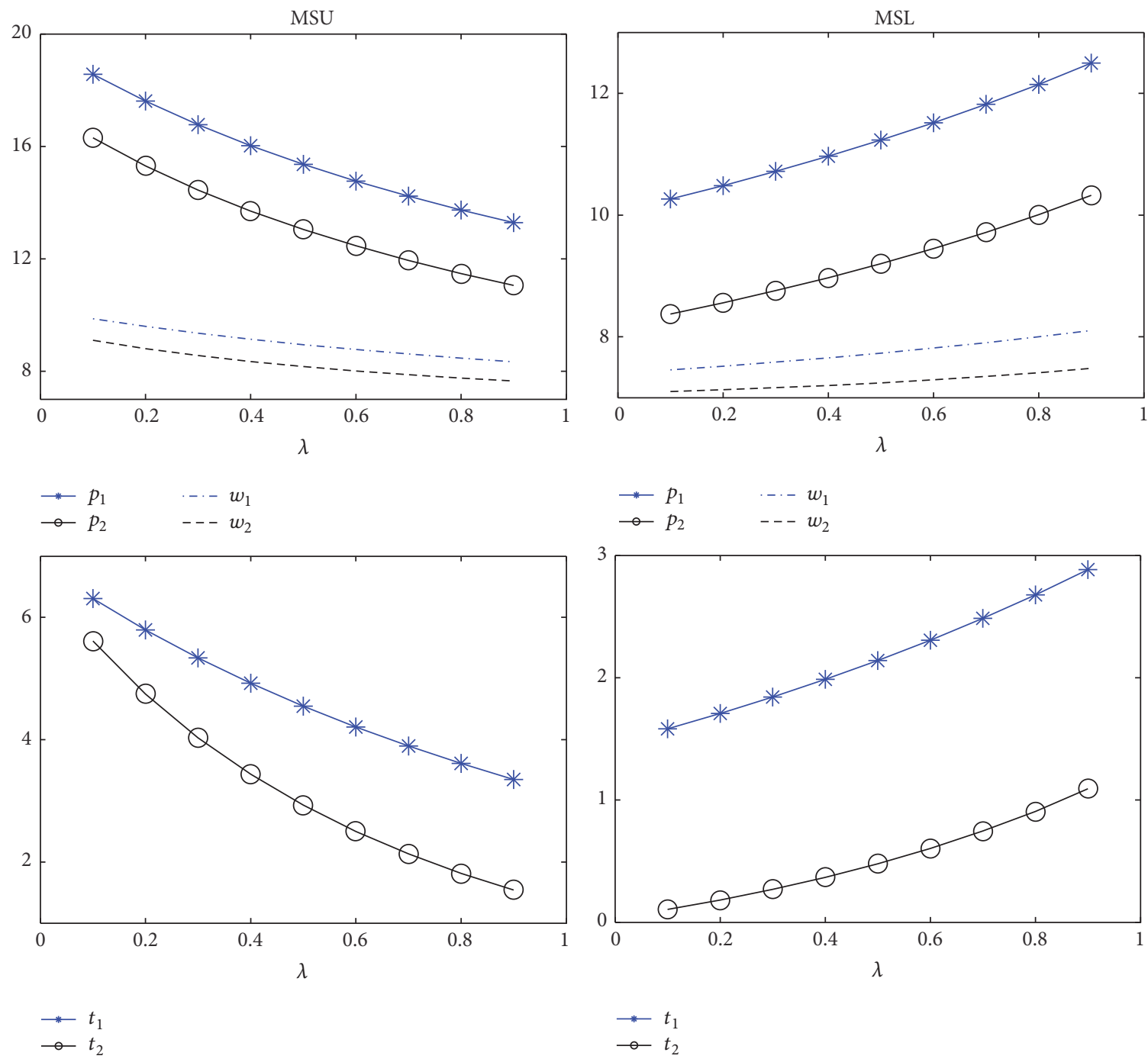

FIGURE 1: Changes of prices and warranty levels with $\lambda$ in MSU and MSL model.

warranty for the retailer, and (c) two manufacturers' warranty services are considered in the supply chain, we compare the $\lambda$-optimistic Stackelberg equilibrium solutions in this subsection. The corresponding results are shown in Tables 5 and 6.

(2-1) The optimal prices and warranty levels under the condition of considering two manufacturers' warranty services are larger than the other scenarios. The firms' maximal profits under the condition of considering two manufacturers' warranty services are higher than the other scenarios. That is because the warranty provided by the manufacturers will stimulate consumer's purchase desire and facilitate final purchase behavior. It would be beneficial for the whole supply chain when the warranty services are introduced into the market.

(2-2) If just manufacturer $m_{1}$ provides the warranty in the market, product 1's optimal pricing decisions increase obviously than that when there is no warranty. On the contrary, product 1's optimal wholesale prices and retail prices decrease more clearly than that when there is no warranty. The firm
TABLE 5: Comparison of the optimal decisions with and without warranty level.

\begin{tabular}{lcccccc}
\hline Scenario & $w_{1}^{*}$ & $w_{2}^{*}$ & $t_{1}^{*}$ & $t_{2}^{*}$ & $p_{1}^{*}$ & $p_{2}^{*}$ \\
\hline $\begin{array}{l}\text { No warranty } \\
\text { level }\end{array}$ & 8.021 & 7.564 & - & - & 12.916 & 10.993 \\
$\begin{array}{l}\text { One warranty } \\
\text { level }\end{array}$ & 8.408 & 7.226 & 3.579 & - & 13.448 & 10.562 \\
$\begin{array}{l}\text { Two warranty } \\
\text { levels }\end{array}$ & 8.467 & 7.755 & 3.611 & 1.815 & 13.735 & 11.474 \\
\hline
\end{tabular}

which provides the warranty service has the advantage in holding more dominant position than without the warranty.

(2-3) In comparison of no warranty and one warranty level in Table 5, it can be known that the wholesale price of product 1 , on account of provision of warranty services, increased by 0.377 , which raised the retailer's purchasing cost. However, the improvement of the retail price ensures the increase in profits. In this case, product 2 had to reduce the 



FIGURE 2: Changes of firms' maximal profits with $\lambda$ in MSU and MSL model.

TABLE 6: Comparison of the maximal profits with and without warranty level.

\begin{tabular}{lcccc}
\hline Scenario & $\Pi_{m_{1}}$ & $\Pi_{m_{2}}$ & $\Pi_{r}$ & $\Pi_{t}$ \\
\hline No warranty level & 3792.9 & 304.8 & 3577.2 & 7674.9 \\
One warranty level & 3971.9 & 199.3 & 3730.2 & 7901.4 \\
Two warranty levels & 4042.4 & 328.2 & 4154.5 & 8525.1 \\
\hline
\end{tabular}

wholesale price to ensure the market share because of no warranty service, and the decline in market competitiveness has led to lower retail prices and lower profits. As is shown in Table 6, it is not difficult to find that the market share of product 1 increased from $92.6 \%$ in the case of no warranty level to $95.2 \%$ in the case of one warranty level, and the profits of retailer and the entire supply chain have been significantly improved. In conclusion, the firm which provides the warranty service has the advantage in holding more dominant position, and it would be beneficial for the whole supply chain when the warranty services are introduced into the market.

Discussion 3 (sensitivity analysis of parameter $\lambda$ ). Numerical studies will be used to illustrate the effects of parameter $\lambda$ on the optimal decisions in MSU and MSL models. By varying the value of $\lambda$ (from 0.1 to 0.9 ), the changes of the optimal decisions can be obtained, which is shown in Figures 1 and 2.

(3-1) It follows from Figures 1 and 2 that the optimal wholesale prices, the optimal warranty levels, the optimal retail prices, the maximal profits of the manufacturer $m_{1}$ and the retailer decrease obviously as $\lambda$ increases in the MSU model and, conversely, increase clearly as $\lambda$ increases in the MSL model. This is consistent with our intuition that the larger the risk resulting from the given confidence level, the more the benefit to the firm.

\section{Conclusion}

Based on game theory and fuzzy theory, we analyze the price and warranty decisions for two substitutable products in a fuzzy supply chain, where the consumer demand, manufacturing costs, and warranty costs for each product are characterized as fuzzy variables. Considering the manufacturers behaving as the Stackelberg leader, we establish expected value model and two chance-constrained programming models. The closed form solutions in each scenario have been proved. Some important analyses about the results are obtained which present managerial insights into the economic behavior of firms and can be afforded as the basis for empirical study in the future, such as the following: (1) the total profits of the two manufacturers are higher than that of the retailer's; this is because the manufacturers play the leader's role which holds the advantage in acquiring more profits in this paper. (2) The warranty provided by the manufacturers will stimulate consumer's purchase desire and facilitate final purchase behavior. It would be beneficial for the whole supply chain when the warranty services are introduced into the market.

Several extensions to this article are possible. First, this paper just considers the scenario where the manufacturers behave as the Stackelberg leader. Further research can extend the models to include Retailer-leader Stackelberg and Nash game models. Second, we establish the models under assuming the symmetry information. Information asymmetry, such as cost and demand information, will be considered in 
the future. Otherwise, more general forms of the demand function can be used to analyze the problem except the linear form.

\section{Competing Interests}

The author declares no conflict of interests.

\section{Acknowledgments}

The support of the Key Scientific Research Projects of Colleges and Universities of Henan Province (nos. 16A130004 and 16A110028) and the research and cultivation fund of Anyang Normal University (no. AYNU-KP-B10) are gratefully acknowledged.

\section{References}

[1] J. Wei, J. Zhao, and Y. Li, "Price and warranty period decisions for complementary products with horizontal firms' cooperation/noncooperation strategies," Journal of Cleaner Production, vol. 105, pp. 86-102, 2015.

[2] W. Kimble and R. O. Lesher, Products Liability, West Publishing Company, St. Paul, Minn, USA, 1979.

[3] M. Van Marrewijk, "Concepts and definitions of CSR and corporate sustainability: between agency and communion," Journal of Business Ethics, vol. 44, no. 2, pp. 95-105, 2003.

[4] P. Ahi and C. Searcy, "A comparative literature analysis of definitions for green and sustainable supply chain management," Journal of Cleaner Production, vol. 52, pp. 329-341, 2013.

[5] K. Jeyakumar, A. Krishnaveni, T. Paul Robert, and D. Jebakani, "Investigation of price, warranty length and production quantity on profit under free replacement warranty policy," Quality Technology \& Quantitative Management, vol. 13, no. 4, pp. 403415, 2016.

[6] M. Esmaeili, N. Shamsi Gamchi, and E. Asgharizadeh, "Threelevel warranty service contract among manufacturer, agent and customer: a game-theoretical approach," European Journal of Operational Research, vol. 239, no. 1, pp. 177-186, 2014.

[7] C. J. Chung and H.-M. Wee, "An integrated productioninventory deteriorating model for pricing policy considering imperfect production, inspection planning and warrantyperiod- and stock-level-dependant demand," International Journal of Systems Science, vol. 39, no. 8, pp. 823-837, 2008.

[8] C.-C. Wu, C.-Y. Chou, and C. Huang, "Optimal price, warranty length and production rate for free replacement policy in the static demand market," Omega, vol. 37, no. 1, pp. 29-39, 2009.

[9] X. Chen, L. Li, and M. Zhou, "Manufacturer's pricing strategy for supply chain with warranty period-dependent demand," Omega, vol. 40, no. 6, pp. 807-816, 2012.

[10] P.-C. Lin and L.-Y. Shue, "Application of optimal control theory to product pricing and warranty with free replacement under the influence of basic lifetime distributions," Computers and Industrial Engineering, vol. 48, no. 1, pp. 69-82, 2005.

[11] C.-C. Wu, P.-C. Lin, and C.-Y. Chou, "Determination of price and warranty length for a normal lifetime distributed product," International Journal of Production Economics, vol. 102, no. 1, pp. 95-107, 2006.

[12] Y.-C. Tsao, W.-G. Teng, R.-S. Chen, and W.-Y. Chou, "Pricing and inventory policies for Hi-tech products under replacement warranty," International Journal of Systems Science, vol. 45, no. 6, pp. 1255-1267, 2014.

[13] Y.-S. Huang, W.-Y. Gau, and J.-W. Ho, "Cost analysis of twodimensional warranty for products with periodic preventive maintenance," Reliability Engineering and System Safety, vol. 134, pp. 51-58, 2015.

[14] J. Zhao, W. Liu, and J. Wei, "Competition under manufacturer service and price in fuzzy environments," Knowledge-Based Systems, vol. 50, pp. 121-133, 2013.

[15] I. P. Krommyda, K. Skouri, and I. Konstantaras, "Optimal ordering quantities for substitutable products with stock-dependent demand," Applied Mathematical Modelling, vol. 39, no. 1, pp.147164, 2015.

[16] B. Liu and R. Zhang, "Joint decisions on pricing and advertising of dominant manufacturer with different advantage strategies," International Journal of Modelling and Simulation, vol. 36, no. 3, pp. 97-105, 2016.

[17] C. Zhou, R. Zhao, and W. Tang, "Two-echelon supply chain games in a fuzzy environment," Computers \& Industrial Engineering, vol. 55, no. 2, pp. 390-405, 2008.

[18] J. Zhao, J. Wei, and Y. Li, "Pricing decisions of complementary products in a two-level fuzzy supply chain," International Journal of Production Research, 2016.

[19] F. Soleimani, "Optimal pricing decisions in a fuzzy dual-channel supply chain," Soft Computing, vol. 20, no. 2, pp. 689-696, 2016.

[20] D. Dubois and H. Prade, Possibility Theory: An Approach to Computerized Processing of Uncertainty, Plenum Press, New York, NY, USA, 1988.

[21] B. Liu, Theory and Practice of Uncertain Programming, Physica, Heidelberg, Germany, 2002.

[22] B. Liu, "A survey of credibility theory," Fuzzy Optimization and Decision Making, vol. 5, no. 4, pp. 387-408, 2006.

[23] Q. Song, K. Shi, S. Lin, and G. Xu, "Optimal decisions for a fuzzy two-echelon supply chain," Discrete Dynamics in Nature and Society, vol. 2014, Article ID 705839, 8 pages, 2014.

[24] J. Zhao and L. Wang, "Pricing and retail service decisions in fuzzy uncertainty environments," Applied Mathematics and Computation, vol. 250, pp. 580-592, 2015.

[25] J. Zhao and J. Wei, "The coordinating contracts for a fuzzy supply chain with effort and price dependent demand," Applied Mathematical Modelling, vol. 38, no. 9-10, pp. 2476-2489, 2014.

[26] D. Chakraborty, D. K. Jana, and T. K. Roy, "Multi-item integrated supply chain model for deteriorating items with stock dependent demand under fuzzy random and bifuzzy environments," Computers and Industrial Engineering, vol. 88, pp. 166-180, 2015.

[27] C. Guo and Z. Liu, "The collaboration and pricing decision of reverse supply chainunder uncertainty," Mathematics in Practice and Theory, vol. 23, no. 39, pp. 27-35, 2009.

[28] J. Wei and J. Zhao, "Pricing decisions with retail competition in a fuzzy closed-loop supply chain," Expert Systems with Applications, vol. 38, no. 9, pp. 11209-11216, 2011.

[29] J. Zhao, W. Tang, and J. Wei, "Pricing decision for substitutable products with retail competition in a fuzzy environment," International Journal of Production Economics, vol. 135, no. 1, pp. 144-153, 2012.

[30] J. Wei, G. Pang, Y. Liu, and Q. Ma, "Pricing decisions of a twoechelon supply chain in fuzzy environment," Discrete Dynamics in Nature and Society, vol. 2013, Article ID 971504, 11 pages, 2013.

[31] L. Wang, J. Zhao, and J. Wei, "Pricing decisions of two complementary products in a fuzzy environment," Mathematical 
Problems in Engineering, vol. 2014, Article ID 729287, 8 pages, 2014.

[32] Q. Gu and J. Ji, "Price decision for reverse supply chain based on fuzzy recycling price," Information and Control, vol. 4, pp. 417-422, 2006.

[33] L.-S. Wang and J. Zhao, "Differential pricing under trading in old goods for new based on market segmentation," in Proceedings of the 31st Chinese Control Conference (CCC '12), pp. 7690-7695, Hefei, China, July 2012.

[34] T. Xiao and D. Yang, "Price and service competition of supply chains with risk-averse retailers under demand uncertainty," International Journal of Production Economics, vol. 114, no. 1, pp. 187-200, 2008. 


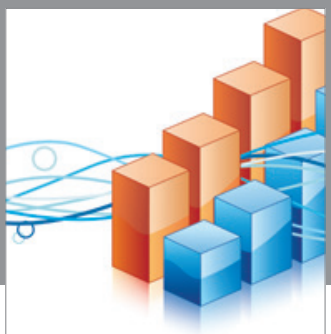

Advances in

Operations Research

vatem alat4

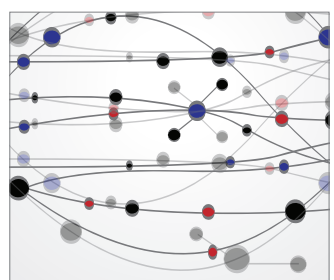

\section{The Scientific} World Journal
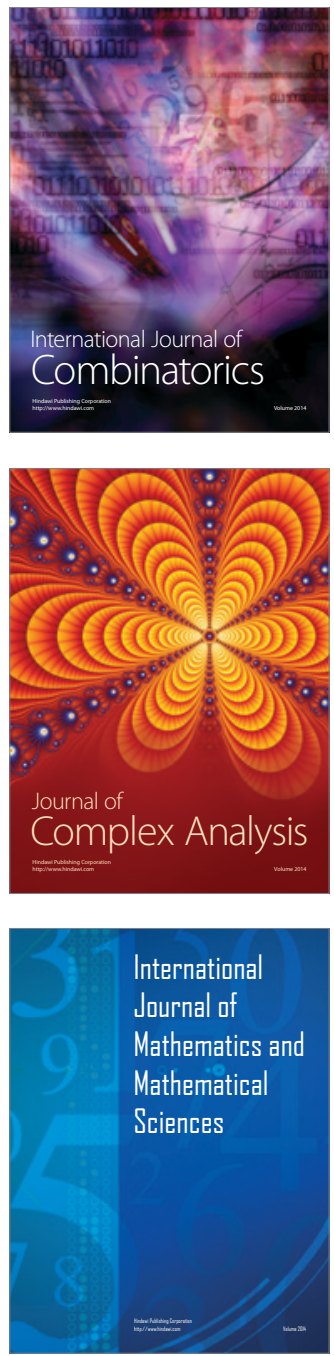
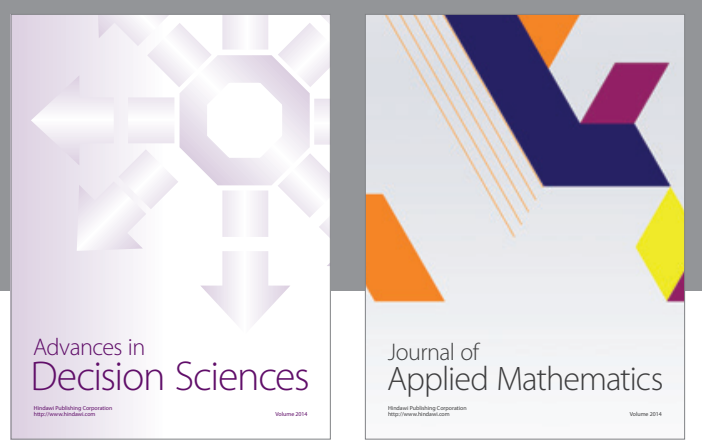

Algebra

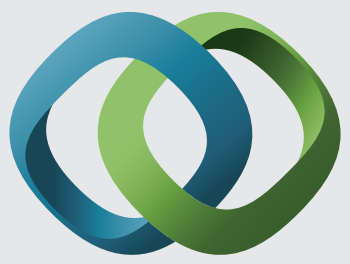

\section{Hindawi}

Submit your manuscripts at

https://www.hindawi.com


Mathematical Problems in Engineering
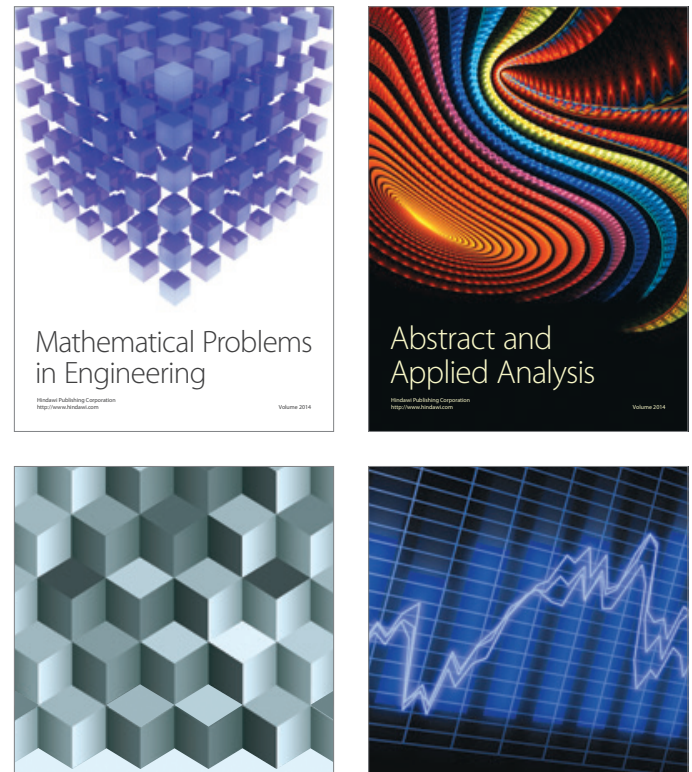

Journal of

Function Spaces



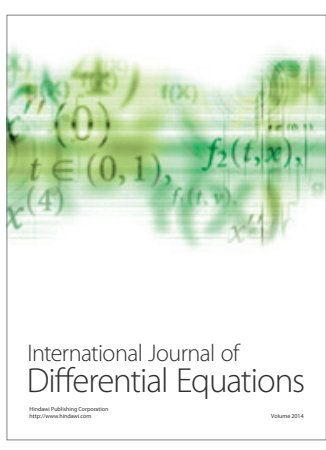
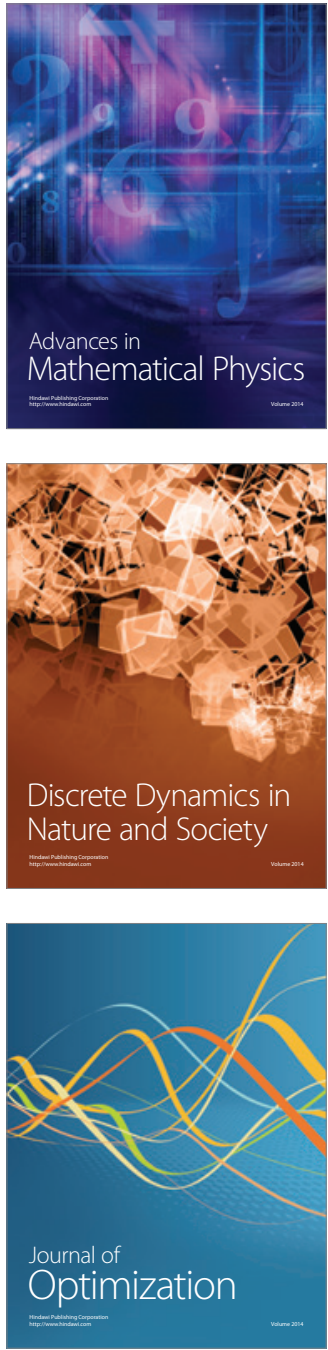